occasionally released into the stomach and causes relaxation of the lower oesophageal sphincter and heartburn, the capsules should not be prescribed with meals or to patients with achlorhydria.

Peppermint oil in enteric-coated capsules appears to be an effective and safe preparation for symptomatic treatment of the irritable bowel syndrome.

We are grateful for advice on and help with enteric-coating procedures from Mr Brian Jones of the technical services division of Eli Lilly and Co Ltd.

${ }^{1}$ Ivey, K J, Gastroenterology, 1975, 68, 1300.

${ }^{2}$ Ritchie, J A, and Truelove, S C, British Medical fournal, 1979, 1, 376.

${ }^{3}$ Evans, B K, et al, Fournal of Pharmacy and Pharmacology, 1975, 27, suppl, p 66.

${ }^{4}$ Rhodes, J B, Abrams, J H, and Manning, R T, Gastroenterology, 1973, $64,829$.

(Accepted 7 August 1979)

University Department of Medicine, Hope Hospital, Manchester M6 8HD

W D W REES, MD, MRCP, Wellcome research fellow

Departments of Gastroenterology and Pharmacy, University Hospital of Wales, Cardiff CF4 4XW

B K EVANS, BPHARM, MPS, principal pharmacist

J RHODES, MD, FRCP, consultant gastroenterologist

\section{Drug names that look or sound alike}

We have compiled the following list of drug names that look or sound alike from reports by our pharmacy staff and by searching through journals.

\begin{tabular}{|c|c|c|c|}
\hline Acetazolamide & Acetohexamide & "Cafergot" & "'Effergot" \\
\hline Acetohexamide & Acetazolamide & "Candeptin" & "Canesten" \\
\hline "Achromycin", & "Aureomycin" & "Canesten", & "Candeptin" \\
\hline "Acriflex" & "Anaflex" & "Carbrital" & "Carbromal" \\
\hline "Actidil" & "Actifed" & "Carbrital" & "Limbitrol" \\
\hline "Actifed" & "Actidil" & "Carbromal" & "Carbrital" \\
\hline "Actifed" & "Linctifed" & "Ceporex" & "Ceporin" \\
\hline "Aldactide", & "Aldactone" & "Ceporin" & "Ceporex" \\
\hline "Aldactone", & "Aldactide", & "Ceporin" & "Cetiprin" \\
\hline Amitriptyline & Nortriptyline & "Cetiprin" & "Ceporin" \\
\hline "Anaflex" & "Acriflex" & "Cetiprin" & "Septrin" \\
\hline "Anovlar", & "Minovlar" & Chlorpromazine & Chlorpropamide \\
\hline "Anthisan" & "Antistin" & Chlorpropamide & Chlorpromazine \\
\hline "Anthisan" & "Anturan", & Clindamycin & "Clinimycin" \\
\hline "Antistin" & "Anthisan" & "Clinimycin", & Clindamycin \\
\hline "Antoin" & "Artane" & "Clomid" & "Atromid" \\
\hline "Anturan" & "Anthisan" & Clomiphene & Clomipramine \\
\hline "Aramine" & "Avomine" & Clomiphene & Clonidine \\
\hline "Artane" & "Antoin" & Clomipramine & Clomiphene \\
\hline "Asilone" & "Ilosone" & Clonidine & Clomiphene \\
\hline "Atarax" & "Ativan" & Clotrimazole & Cotrimazine \\
\hline "Ativan" & "Atarax" & Clotrimazole & Co-trimoxazole \\
\hline "Atromid" & "Clomid" & "Codis tabs" & "Cortistab" \\
\hline "Aureomycin" & "Achromycin", & "Corlan" & "Cortelan" \\
\hline "Avomine" & "Aramine" & "Cortelan" & "Corlan" \\
\hline "Reconace", & & $\begin{array}{l}\text { "Cortisyl" } \\
\text { "Cortistab", }\end{array}$ & $\begin{array}{l}\text { "Cortril" } \\
\text { "Codis tabs", }\end{array}$ \\
\hline "Becotide", & $\begin{array}{l}\text { "Becotide" } \\
\text { "Beconase", }\end{array}$ & $\begin{array}{l}\text { "Cortistab" } \\
\text { "Cortril"" }\end{array}$ & "Cortisyl" \\
\hline "Benemid" & "Benuride", & Co-trimazine & Co-trimoxazole \\
\hline "Benoral" & "Benoxyl", & Co-trimoxazole & Clotrimazole \\
\hline "Benoxyl", & "Benoral", & Co-trimoxazole & Co-trimazine \\
\hline "Benuride" & "Benemid" & "Cytacon", & "Cytamen" \\
\hline Bethanecol & Bethanidine & "Cytamen", & "Cytacon" \\
\hline Bethanidine & Bethanecol & & \\
\hline "Betnelan"" & "Betnesol" & & \\
\hline "Betnesol", & "Betnelan", & "Danol", & "Daonil" \\
\hline "Betnesol", & "Bextasol", & "Daonil" & "Danol" \\
\hline "Bextasol"' & "Betnesol", & "Dehydrocholin", & Dihydrocodeine \\
\hline Biogastrone" & "Duogastrone" & "Demulen" & "Femulen" \\
\hline "Bradosol", & "Brasivol", & "Deseril" & "Desferal" \\
\hline "Brasivol", & "Bradosol"' & "Desferal" & "Deseril"' \\
\hline "Butacote", & "Butamet", & "Dexedrine" & "Dexamed" \\
\hline "Butomet" & "Butacote", & "Dexamed" & "Dexedrine" \\
\hline "Butazolidin", & "Parazolidin" & "Diabinese" & "Glibenese" \\
\hline
\end{tabular}

Digitoxin Digoxin

Digoxin

Dihydrocodeine

"Dimotane"

"Dimotane LA"

"Dimotapp"

Dipyridamole

Disopyramide

"Donnatal LA"

Dothiepin

Doxepin

"Duogastrone"

"Durabolin"

"Duromine"

"Durenate"

"Dytac"

"Dytide"

"Effergot"

Ergometrine

Ergotamine

Ethinyloestradio

Ethyloestrenol

"Femergin"

"Femulen"

"Fucidin"

"Fucidin"

"Fulcin"

"Furacin",

"Gardenal"

"Gefarnil"

Glibenclamide

"Glibenese"

Glibornuride

Hydroxyzine hydrochloride

Hydroxyzine

pamoate

"Ilosone"

Imipramine

"Imperacin"

"Keflex"

"Keflin"

"Kemadrin"

"Kinidin"

"Lederspan"

"Lergoban"

Levallorphan

Levorphanol

"Limbitrol"

"Linctifed"

"Magmilor"

"Megaclor"

"Mestinon"

"Mesontoin"

Methenamine

Methionine

Digitoxin

"Dehydrocholin"

"Dimotapp"

"Donnatal LA"

"Dimotane"

Disopyramide

Dipyridamole

"Dimotane LA"

Doxepin

"Dothiepin"

"Biogastrone"

"Orabolin"

"Durenate"

"Duromine"

"Dytide"

"Dytac"

"Cafergot"

Ergotamine

Ergometrine

Ethyloestrenol

Ethinyloestradiol

"Phenergan"

"Demulen"

"Fulcin"

"Furacin"

"Fucidin"

"Fucidin"

"Gefarnil"

"Gardenal"

Glibornuride

"Diabenese"

Glibenclamide

Hydroxyzine

pamoate

Hydroxyzine

hydrochloride

"Asilone"

"Imperacin"

Imipramine

"Keflin",

"Keflex"

"Kinidin"

"Kemadrin"

"Lergoban"

"Lederspan"

Levorphanol

Levallorphan

"Carbrital"

"Actifed"

"Midamor"

"Mogadon"

"Mesontoin"

"Mestinon"

Methionine

Methenamine

Proprietary names are given in quotation marks.

\section{Comment}

We do not claim that this list is comprehensive, but we think it will be useful to any doctor prescribing. We welcome any additions.

(Accepted 16 August 1979)

Regional Drug Information Centre, Bristol Royal Infirmary, Bristol BS2 8HW

H MCNULTY, PHD, BPHARM, principal pharmacist

Pharmacy Department, Bristol Royal Infirmary, Bristol BS2 8HW P SPURR, BPHARM, MPS, staff pharmacist

"Magmilor"

"Naxogin"

"Megaclor",

"Movelat"

"Motival"

"Mixogen"

Nicotinic acid

Nicotinamide

Amitriptyline

"Nuelin"

"Durabolin"

"Butazolidin"

"Priadel"

Penicillin

Penicillamine

Phenobarbitone

"Femergin"

Pentobarbitone

"Ponstan"

"Piriton"

Perhexiline

"Parlodel"

Promethazine

Quinine

Quinidine

"Rifinah"

"Rifadin"

"Sando-K"

"Sandocal"

"Zarontin"

"Serenid"

"Serenace"

"Sintisone"

"Sinthrome"

Sulphamethizole

"Septrin"

"Torecan"

"Teronac"

Trifluoperazin

Trimeprazine

"Saroten" 